\title{
Caracterización de la flora arbórea de algunos bosques delimitados en la zona costera de los distritos Bahía Ballena y Puerto Cortés
}

\section{Characterization of the arboreal flora of some forests delimited in the coastal zone of the Bahía Ballena and Puerto Cortés districts}

\author{
Juan Carlos Villegas-Arguedas ${ }^{1}$ \\ Sistema Nacional de Áreas de Conservación Costa Rica
}

\begin{abstract}
Resumen. Se estudió las estructuras de algunos bosques costeros de Osa en la zona sur de Costa Rica, en vertical a partir de las especies y sus alturas $(\mathrm{H})$ por individuos y horizontal determinada por las especies, sus diámetros a la altura del pecho (DAP>15 cm), área basal $(\mathrm{AB})$, volumen $(\mathrm{V})$, además del cálculo de un Índice de Valor de Importancia (IVI) a partir de su frecuencia, abundancia y dominancia, lo que permitió su caracterización. De mayo a diciembre 2016 y febrero hasta abril 2017, se ubicaron parcelas demostrativas para la identificación de especies; las parcelas demostrativas fueron circulares equidistantes de $r=12.62 \approx a=500 \mathrm{~m}^{2}$, separadas una de otra $50 \mathrm{~m}$ hasta un máximo de parcelas permitido por el límite extremo del polígono de bosque en estudio. Se identificó 1787 individuos de 112 especies de árboles, 1363 corresponden a Bahía Ballena, mientras que 424 a Puerto Cortés. Se registró un promedio de 229 Ind/ha en toda la zona de estudio, en los bosques del distrito de Bahía Ballena se registró un promedio de 218.08 Ind/ha, mientras que en el distrito de Puerto Cortés en promedio se registró 273.5 Ind/ha. Las especies más comunes fueron Inga vera, Guazuma ulmifolia, la Vochysia guatemalensis e Inga punctata; la diversidad total $\mathrm{H}^{\prime}$ fue de 3.88 según Shannon-Wiener. A partir de los registros y análisis desarrollados, se concluye que la principal diversidad de bosques y elementos arbóreos de la biodiversidad se encuentran en el distrito de Bahía Ballena; no así en Puerto Cortés, donde estos recursos forestales son en la actualidad escasos.
\end{abstract}

Palabras clave. Bosque, diversidad, Bahía Ballena, Puerto Cortés, Costa Rica.

\begin{abstract}
We studied the structures of some coastal forests of Osa in the south zone of Costa Rica in vertical from the species and their heights $(\mathrm{H})$ by individuals and horizontal determined by the species, their diameters at breast height (DAP> $15 \mathrm{~cm}$ ), basal area (AB), volume (V), in addition to the calculation of a Value Index of Importance (IVI) from its frequency, abundance and dominance, which allowed its characterization. Demonstration plots for the identification of species were located from May to December 2016 and February to April 2017; the demonstration plots were equidistant circular plots of $\mathrm{r}=12.62 \approx \mathrm{a}=500 \mathrm{~m}^{2}$ separated one from another $50 \mathrm{~m}$ up to a maximum of plots allowed by the extreme boundary of the forest polygon under study. We identified 1787 individuals from 112 tree species, 1363 from Bahía Ballena, and 424 from Puerto Cortés. An average of 229 Ind / ha was recorded in the entire study area; in the forests of the Bahía Ballena district, an average of 218.08 Ind / ha was recorded, while in the Puerto Cortés district an average of 273.5 Ind / ha was recorded. The most common species were Inga vera, Guazuma ulmifolia, Vochysia guatemalensis and Inga punctata; the total diversity $\mathrm{H}^{\prime}$ was 3.88 according to Shannon-Wiener. From the records and analyzes developed, it is concluded that the greatest diversity of forests and arboreal elements of biodiversity are found in the district of Bahía Ballena, not so in Puerto Cortés, where these forest resources are currently scarce.
\end{abstract}

Keywords. Forest, Diversity, Ballena Bay, Puerto Cortés, Costa Rica.

'Juan Carlos Villegas-Arguedas. Sistema Nacional de Áreas de Conservación, Departamento de Prevención, Protección y Control. Doctorando en Ciencias Naturales para el Desarrollo, Costa Rica. Dirección electrónica: juan.villegas@sinac.go.cr 


\section{Introducción}

El conocimiento de la integridad y función ecológica de los bosques parte de su conocimiento estructural, lo cual a su vez repercute en las propuestas para su manejo exitoso. Esto es fundamental dada la reducción continua e impactos ambientales negativos a los que los bosques tropicales han sido sometidos (Cascante y Estrada, 2001; Sánchez et al.2005).

En Costa Rica, los bosques fuera de las grandes áreas protegidas se encuentran dispersos en parches de distintos tamaños y a distancias variables entre estos, particularmente asociados a las zonas de protección de ríos y quebradas o en mosaicos ecosistémicos agrícolas donde los parches son aún más distantes entre ellos, debido a las grandes extensiones de terrenos para la agricultura o la ganadería (Tenorio, Solano-Durán y Castillo-Ugalde, 2009; MoralesSalazar et al. 2012); son, por consiguiente, vestigios boscosos de gran vulnerabilidad (Vargas-Fonseca, 2012; Dirzo et al. 2014; Morera, Sandoval y Pintó, 2018).

De acuerdo con el último inventario exhaustivo de la cobertura forestal de Costa Rica, el país cuenta con 3866895.8 ha de bosques, lo cual representa $75.5 \%$ de cobertura vegetal en el territorio nacional. La superficie no forestal es de 773135.2 ha. Del 15.1\%, del área con cobertura forestal, corresponde a bosque maduro $40.05 \%$; asimismo, $24.33 \%$ son bosques secundarios y $31.54 \%$ son pastos con árboles, entre las principales coberturas (SINAC 2015).

Diversas son las variables ambientales que determinan la estructura y composición de los bosques tropicales. Se consideran aspectos bioclimáticos de temperatura, altitud, precipitación o tipos de suelos, o variables biológicas como la polinización y dispersión, condiciones que determinan no solo su conservación de éstos sino su capacidad regenerativa y de adaptación (Leiva, Rocha, Mata y Gutiérrez-Soto, 2009; Celentano et al. 2011).

Esta investigación cumplió como objetivo conocer la estructura horizontal y vertical de algunos parches boscosos identificados y delimitados en el área de estudio para la zona costera de los distritos Bahía Ballena y Puerto Cortés del cantón de Osa, en la zona sur de Costa Rica, lo cual permite su caracterización y como hipótesis se esperó que la mayor diversidad de bosques y su estado de conservación se encuentre en el distrito de Bahía Ballena, dada su mayor cercanía del pie de monte a la línea de costa y su menor presencia de aluviones utilizado para la agricultura extensiva.

Los bosques de las zonas costeras. No obstante, la importancia que se le ha dado a los bosques del neotrópico por su complejidad, biodiversidad, capacidad de carga, germoplasma y captura de carbono, o por su acelerada pérdida por la deforestación, las investigaciones para el conocimiento de su estructura y diversidad se consideran escasos en la región, con aportes puntuales en algunos países y para algunos grupos dendrológicos (Berry, 2002; Barquero y Jiménez, 2009).

Los bosques costeros no solo han sufrido el impacto ambiental negativo por la expansión de la frontera agrícola, fenómeno documentado a partir de la segunda mitad del siglo XX (Armenteras y Rodríguez, 2014), sino que en años recientes las zonas costeras han tenido también la presión por la urbanización turística como incentivo estatal para la generación de empleo en las zonas rurales. Dicho fenómeno provoca más fragmentación de estos ecosistemas (Morera, 2010; Barrantes, 2013).

Costa Rica es de los países de la región centroamericana que posee menos población en las zonas costeras (Windevoxhel, Rodríguez y Lahmann, 2003), lo cual ha provocado un 
descuido estatal por el desarrollo integral de estas zonas, con el consiguiente impacto y fragmentación de estos bosques producto de la falta de control y el desordenado desarrollo.

No obstante algunos esfuerzos por el inventario de remanentes de Patrimonio Natural del Estado en la zona marítimo-terrestre, particularmente en la zona sur (Villegas, 2017), el país no cuenta con una línea base de información que cuantifique las pérdidas de los bosques de las zonas costeras; por lo tanto, los resultados de esta investigación podrían ser utilidad en la toma de decisiones, planes reguladores o de conservación $\mathrm{u}$ otras políticas de manejo ambiental que se implementen en el futuro.

Caracterización de los bosques. La disposición de los parches de bosques en una matriz ecosistémica en la cual se mezclan los elementos naturales con los paisajes antrópicos, afecta negativamente su complejidad estructural y los procesos ecológicos intrínsecos (Sauders, Hobbs \& Margules, 2001; Pincheira-Ulbrich, Rau y PeñaCortés, 2009), lo que hace necesario su estudio periódico y la evaluación del impacto ambiental negativo con propósitos de conservación.

Aspectos como la diversidad, el tamaño de las poblaciones y otros procesos ecológicos como la polinización y dispersión son afectados por la distancia entre parches, su forma y su tamaño, aspectos biogeográficos poco estudiados en comunidades de plantas (Galanes \& Thomlinson, 2009).

Durante el desarrollo de la investigación en ecología, dogmáticamente algunos ecólogos asumieron que el proceso del estudio de bosques fragmentados y su estructura se compara con la biogeografía de islas oceánicas (Harris, 1984; Laurance \& Bierregaard 1997; Rosenblatt et al. 1999; Ferraz et al. 2003; Hill \& Curran, 2003).

Esta comparación presenta una serie de inconsistencias que no la hacen del todo aplicable o puede sugerirse solo en algunas ocasiones, debido a que la matriz de vegetación o el ensamblaje taxonómico arbóreo-arbustivo entre parches introduce una covariable cuyo aporte a la estructura de los parches boscosos aún requiere investigación. Su aporte biológico es aún dudoso (e.g. pastizales arbolados).

También existe una variable tiempo entre los disturbios humanos a los parches o creación de estos respecto a la longevidad de las especies de árboles- Esta repercute en el conocimiento real de la dinámica poblacional de las especies arbóreas; entonces, algunas variables abióticas como el suelo pueden cambiar drásticamente entre parches a nivel regional, lo que a la vez hace difícil la comparación por variables incontroladas que repercuten en la estructura y diversidad entre parches (Cayuela, Golicher, Rey, González-Espinoza \& Ramírez-Marcial, 2006).

En Costa Rica se han desarrollado investigaciones de campo referentes a la biodiversidad de parches boscosos, Así, se ha demostrado su importancia ecológica como sumideros de diversidad y bancos de germoplasma, generadores de microclimas, mantenimiento de fuentes de agua y otros diversos servicios ecosistémicos que aportan ( $\mathrm{Di}$ Stéfano, Nielsen, Hoomans y Fournier, 1996).

El entendimiento de cómo funcionan y se manejan los bosques tropicales, secos o húmedos parte de los inventarios que permitan determinar la complejidad florística y estructural de la masa vegetal, mediante la evaluación de las especies presentes y sus cantidades (Di Stéfano, Brenes y Mora, 1995). Según sea el interés en profundizar su análisis para la estructura horizontal o vertical, se contemplan otras variables como la densidad, frecuencia, dominancia, patrones de distribución y volúmenes de las especies o análisis de alturas y estratos (Lamprecht 1990; Di Stéfano et al., 1995; Hernández, 2000; Oviedo, Alvarado y Fournier, 2009; Morales, 2010). 


\section{Metodología}

La La investigación se desarrolló en la zona costera de los distritos Bahía Ballena y Puerto Cortés hasta el pie de monte, definido en la cota $20 \mathrm{~m}$ s.n.m, para un área de estudio total de 12223.10 hectáreas, entre los $9^{\circ} 16^{\prime}$ Norte, $83^{\circ} 50^{\prime}$ Oeste y los $8^{\circ} 7^{\prime}$ Norte, $83^{\circ} 28^{\prime}$ Oeste (fig. 1).

En razón de la baja elevación y lo angosto del área de muestreo en algunos de sus tramos se consideró homogénea para la variable suelos, que según Kappelle, Castro, Acevedo, González y Monge (2002) corresponden tipo Mollisoles.

Se utilizó la delimitación de bosques de Villegas (2017) donde los bosques corresponden con la mayor cantidad de polígonos identificados $(n=65)$ con un área de cobertura de 639.10 hectáreas; de ellos, 50 polígonos de bosque se ubican en el distrito Bahía Ballena, mientras que 15 en Puerto Cortés (fig. 2).

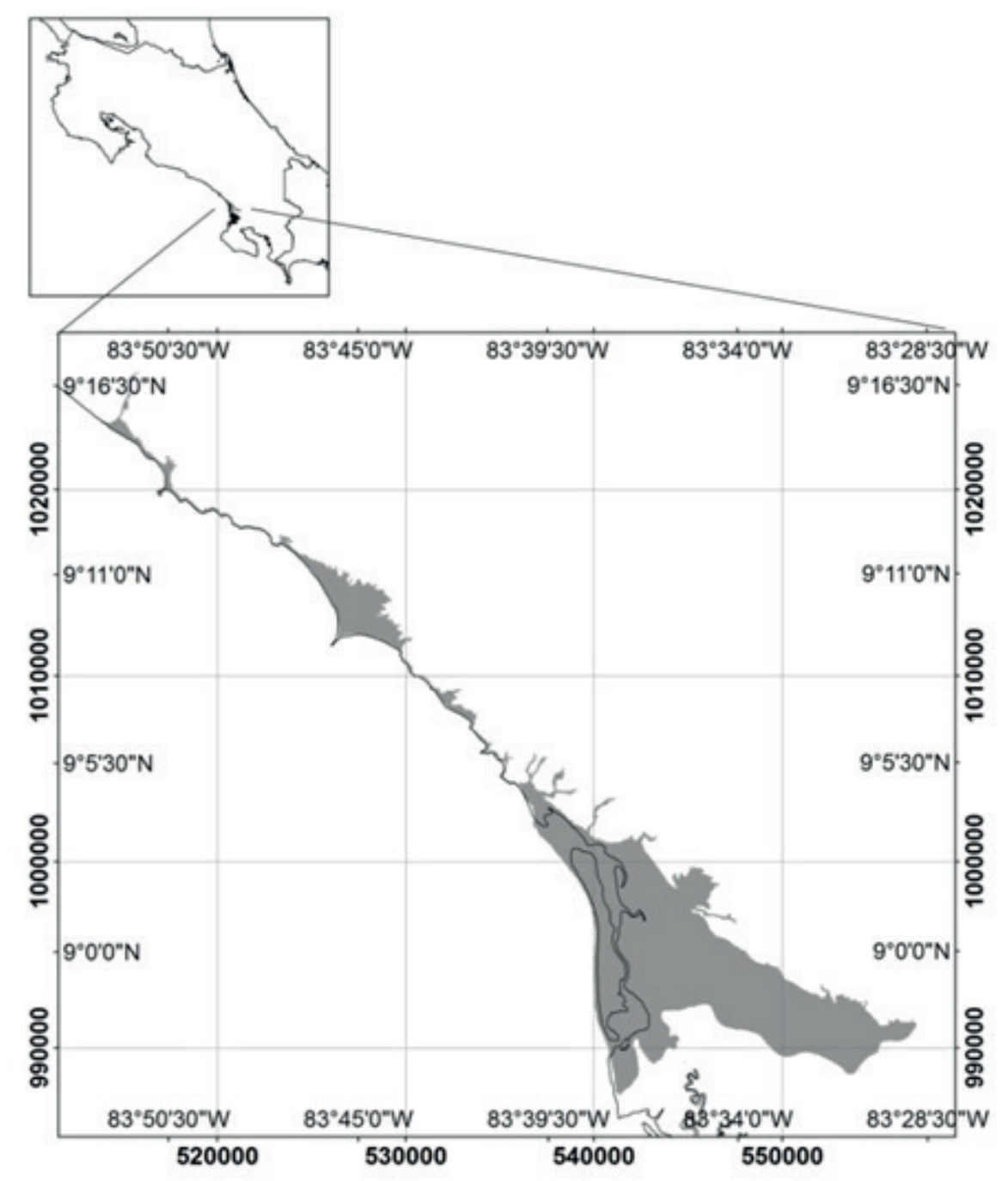

Figura 1. Ubicación del área de estudio. Fuente: Juan Carlos Villegas, 2017 


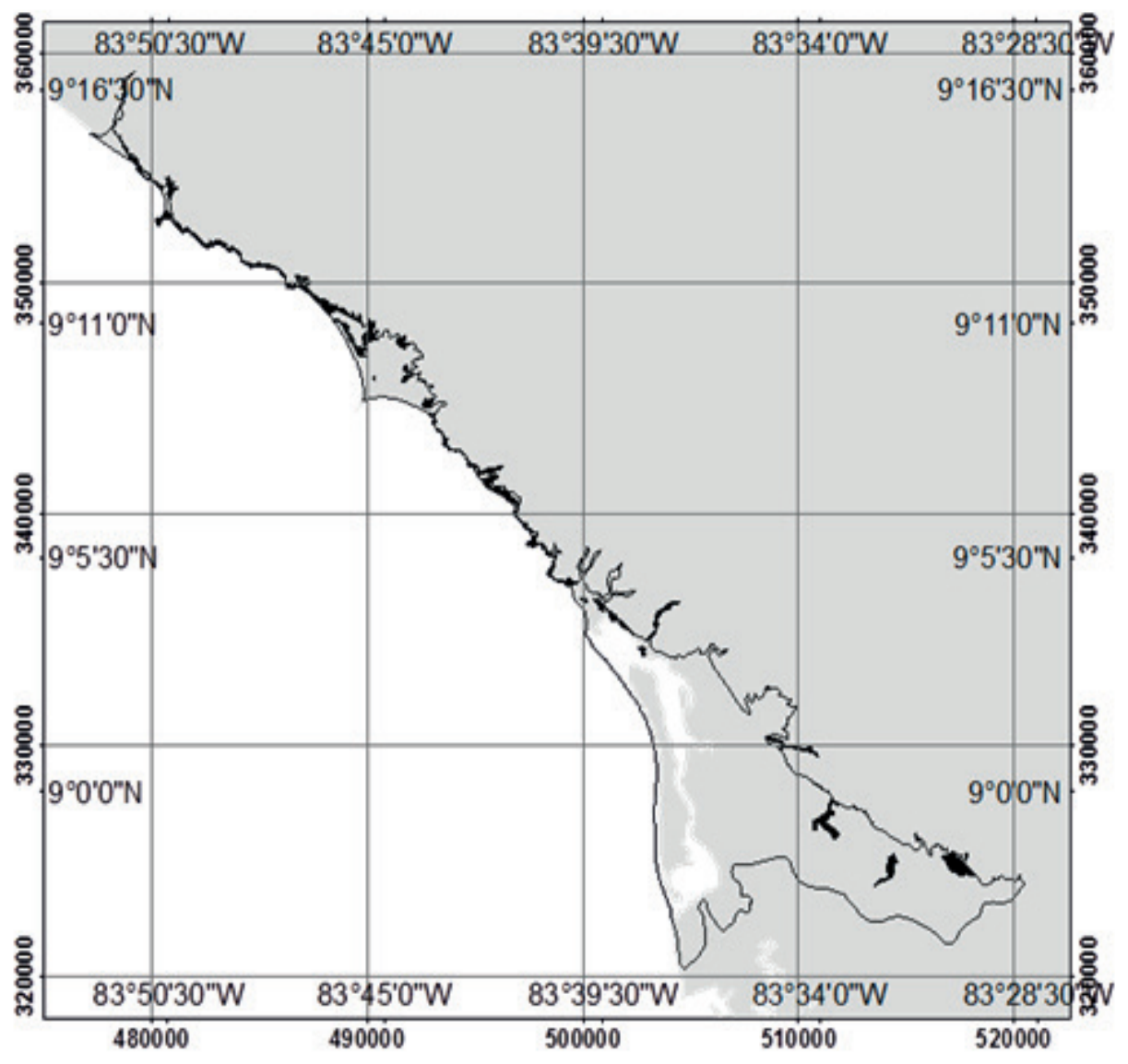

Figura 2. Ubicación de bosques en el área de estudio. Fuente: Juan Carlos Villegas, 2017.

Durante mayo, julio, setiembre, octubre y diciembre 2016, además de febrero y abril 2017, se ubicaron 156 parcelas demostrativas para la identificación de especies de árboles en los parches de bosque preestablecidos. Se consideró sitios con facilidad de acceso donde fue factible su recorrido en sotobosques ralos. Se tomó en cuenta, asimismo, el área total de bosques, con la cantidad de parcelas predefinidas y la intensidad de muestreo fue de $1.22 \%$.

La ubicación de cada parcela de muestreo se georeferenció con un GPS (Global Positional System) marca Garmin, modelo 60Cx (+/- $2 \mathrm{~m})$, y se trasladó a un programa de computo GPS
Utility Ltd. 1998-2008 Versión 4.93 según www. gpsu.co.uk con proyección Lambert Sur para Costa Rica y Datum Nad27 Central y Sistema de Información Geográfica ArcMap 10.1 ${ }^{\mathrm{TM}}$ de ESRI@ (Enviroment Systems Reserch Institute Inc.).

Las parcelas demostrativas fueron circulares equidistantes de $\mathrm{r}=12.62 \approx \mathrm{a}=500 \mathrm{~m}^{2}$ medidas con un telémetro marca Leica Geosystems, modelo Leica Disto ${ }^{\mathrm{TM}} \mathrm{A} 3$. La primera ubicación inició a $25 \mathrm{~m}$ de la línea que delimita el polígono de bosque; luego quedaron separadas unas de otras 50 m hasta un máximo de parcelas que permitió el límite extremo del polígono. 
Se estudió las estructuras vertical y horizontal de estos bosques costeros según:

Estructura vertical: Determinada a partir de las especies y sus alturas $(\mathrm{H})$ por individuos; altura de los árboles mayores de $15 \mathrm{~cm}$, establecida mediante un inclinómetro para lectura óptica marca Suunto, modelo PM-5/1520.

Estructura horizontal: Determinada por las especies, sus diámetros a la altura del pecho $(\mathrm{DAP}>15 \mathrm{~cm})$ medida mediante una cinta diamétrica marca Richter de $5 \mathrm{~m}$, el área basal $(\mathrm{AB})$, volumen $(\mathrm{V})$, además del cálculo de un Índice de Valor de Importancia (IVI) a partir de su frecuencia, abundancia y dominancia (Curtis \& McIntosh, 1951).

La adscripción taxonómica nomenclatural de las especies arbóreas sigue los criterios del Missouri Botanical Garden (2017: http://www. missouribotanicalgarden.org/plant-science/ plant-science/resources.aspx) y se determinó a nivel taxonómico específico mediante las claves de Woodson y Schery (1980) y Hammel, Zamora y Grayum (1998).

Se registraron los datos de campo del inventario de árboles en una hoja de cálculo diseñada para el manejo estadístico de datos y sus tablas dinámicas en Excel 2010 (MicroSoft ${ }^{\circledR}$ ). La diversidad arbórea (conocida como diversidad alfa $\mathrm{H}^{\prime}$ por ser exclusiva de un sitio particular de muestreo) se estableció mediante el índice ecológico de proporcionalidad Shannon-Wiener $\left(\mathrm{H}^{\prime}=-\sum\right.$ pi ln pi) según los criterios de Magurran (1988), Henderson y Seaby (2006) y Moreno (2001). Se aplicó el Random Test de Solow (1993) para determinar la existencia de diferencias estadísticamente significativas entre los distritos.

A fin de determinar la significancia de las diferencias estadísticas en la variable abundancia por distritos, se utilizó un ANOVA no paramétrico U de Mann-Whitney para datos pareados según Sokal y Rohlf (1981) y Fowler,
Cohen y Jarvis (1988) con la ayuda de InfoStat versión 1.1 (Balzarini et al. 2017).

Para inferir el comportamiento del crecimiento de la masa boscosa y determinar una eventual edad y su capacidad de regeneración, se utilizó la distribución de todos los individuos según la clase diamétrica a la que pertenece, graficando según los criterios de Cook, Briffa, Shiyatov, Mazepa y Jones (1992).

Con la finalidad de establecer la calidad del inventario y la fiabilidad de la muestra dendrológica, se realizó una curva de acumulación de especies siguiendo los criterios de Jiménez-Valverde y Hortal (2003).

\section{Resultados}

Se ubicó y georeferenció 156 parcelas demostrativas, de las cuales 125 correspondieron al distrito Bahía Ballena entre Dominical y playa Ventanas, mientras que 31 al distrito de Puerto Cortés entre Tortuga Abajo y Punta Mala de Coronado.

Se identificó 1787 individuos de 112 especies de árboles, tanto nativas como introducidas, de los cuales 1363 ind. corresponden a Bahía Ballena, mientras que se halló 424 se en Puerto Cortés, para un error de muestreo de 3.83\% (tabla 1).

Se registró un promedio de 229 Ind/ha. para toda el área de estudio, con un promedio de 218.08 Ind/ha. en el distrito Bahía Ballena, en tanto que en el distrito de Puerto Cortés el promedio fue de $273.5 \mathrm{Ind} / \mathrm{ha}$.

La zona de estudio presentó alta diversidad arbórea, con diversidad $\mathrm{H}^{\prime}$ alta para ambos distritos, sin sugerir diferencias estadísticamente significativas entre distritos (Random Test Solow $\left.\mathrm{D}=0.55 ; p=0,0001^{* * *}\right)$, los cuales a su vez sugieren que comparten casi en 50\% las especies de árboles en la zona (tabla 1). Estos bosques registraron árboles de baja altura, no superando en promedio los $10 \mathrm{~m}$. 
Tabla 1

Especies forestales identificadas por sector de muestreo en los distritos Bahía Ballena y Puerto Cortés, Osa 2017.

\begin{tabular}{lccc}
\hline Características & \multicolumn{2}{c}{ Distrito } & Total \\
\hline & Bahía Ballena & Puerto Cortés & 112 \\
\cline { 2 - 3 } Número de especies & 102 & 52 & 3.88 \\
Índice de diversidad $\left(\mathrm{H}^{\prime}\right)$ & 3.89 & 3.33 & 1787 \\
Número de individuos & 1363 & 424 & 38.79 \\
DAP promedio & 39.07 & 37.91 & 317.15 \\
Área basal (m2) & 234.14 & 83.01 & 0.46 \\
Índice de similitud (S) & 0.46 & 0.46 & 9.71 \\
Altura dosel promedio & 9.63 & 9.96 & \\
\hline
\end{tabular}

Fuente. Juan Carlos Villegas, 2017

Las especies de árboles que presentaron individuos con alturas superiores fueron la ceiba (Ceiba pentandra, $n=1$ ), la cual registró una altura promedio de $32 \mathrm{~m}$; seguida del árbol terciopelo o pica pica (Sloanea terniflora, $n=1$ ) con $20 \mathrm{~m}$ de altura promedio; al igual que una especie de aguacatillo (Ocotea glaucosericea, $n=1$ ), además del baco (Brosimum utile, $n=6$ ) con un promedio de altura de $18.5 \mathrm{~m}$.

No se registró diferencias estadísticamente significativas para la abundancia arbórea por distritos $\left(\mathrm{U}=783.5 ; p=0.0002^{* * *}\right)$, lo cual sugiere que los bosques en ambos distritos contienen cantidades similares de árboles en su conformación estructural.

La distribución de los individuos por clases diamétricas demuestra una conformación de una "J" invertida (fig. 3). La mayoría $(n=887)$ se ubican en la clase diamétrica inferior (DAP=10$30 \mathrm{~cm})$, mientras que muy pocos individuos $(n=1)$ tiene DAP superiores a los $230 \mathrm{~cm}$.

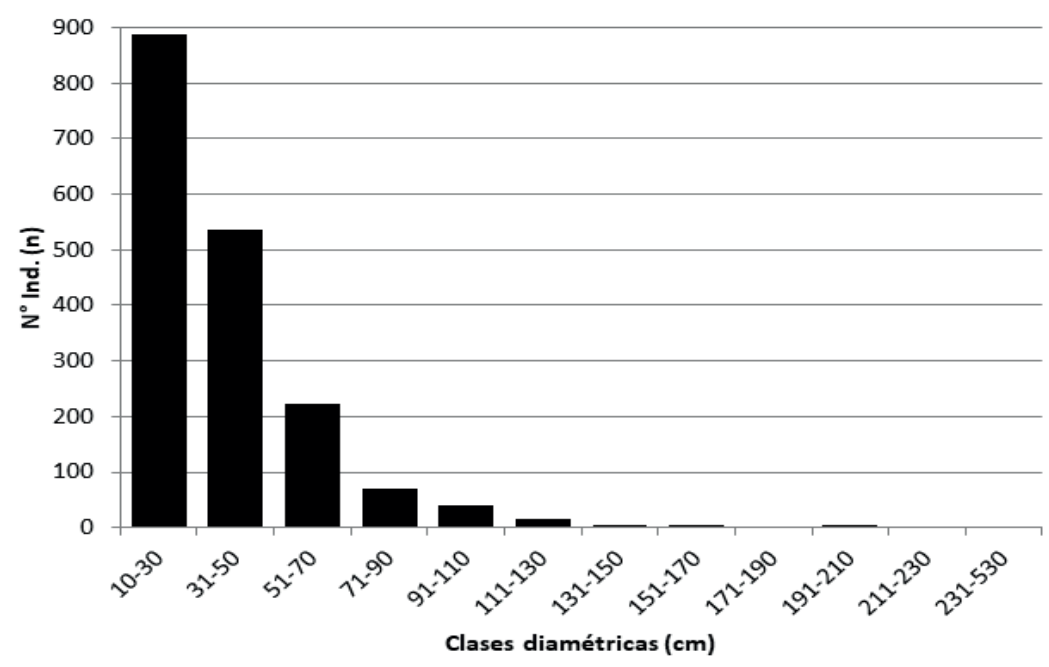

Figura 3. Distribución por clases diamétricas de los individuos determinados en el área de estudio, Osa 2017. Fuente: Juan Carlos Villegas, 2017. 
Las especies más comunes fueron guaba (Inga vera, n=106), guácimo colorado (Guazuma ulmifolia, $n=103)$, árbol de mayo o botarrama (Vochysia guatemalensis, $n=93$ ) y otra especie de guaba (Inga punctata, $n=92$ ) (tabla 2).

Las especies de ceiba (Ceiba pentandra), chilamate (Ficus insípida), gallinazo (Schizolobium parahyba) y balso (Ochroma pyramidale) presentaron mayor área basal y volumen de su madera, con áreas basales de 22.06, 17.93, 14.85 y 17.18, respectivamente, y volúmenes de 705.98, 253.28, 232.24 y 223.72, en el mismo orden.
Al considerar la totalidad de individuos, se registró un área basal de $317.15 \mathrm{~m}^{2}$ y un volumen de $4351.15 \mathrm{~m}^{3}$ de madera. Las especies de más importancia ecológica a partir del respectivo IVI (Índice de Valor de Importancia) son la guaba Inga vera (IVI=16.09); el guácimo colorado (Guazuma ulmifolia; IVI=14.98) y la palma corozo (Elaeis oleífera; IVI=13.91)-Ambos distritos presentaron diferencia en la representación de sus especies importantes (tabla 2).

\section{Tabla 2}

Índice de Valor de Importancia para las principales especies determinadas en el área de estudio, Osa 2017

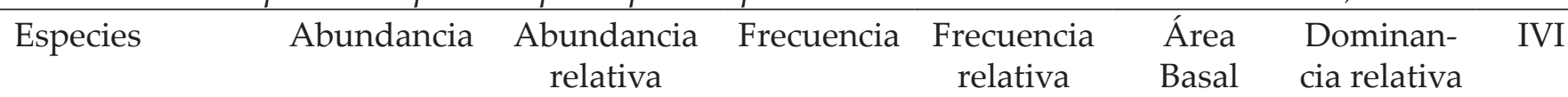

\begin{tabular}{|c|c|c|c|c|c|c|c|c|}
\hline \multicolumn{9}{|c|}{ Todos los sectores } \\
\hline 1 & Inga vera & 106 & 5.93 & 58 & 6.68 & 11.03 & 3.48 & 16.09 \\
\hline 2 & $\begin{array}{l}\text { Guazuma } \\
\text { ulmifolia }\end{array}$ & 103 & 5.76 & 37 & 4.26 & 15.71 & 4.95 & 14.98 \\
\hline 3 & Elaeis oleífera & 87 & 4.87 & 20 & 2.30 & 21.38 & 6.74 & 13.91 \\
\hline 4 & $\begin{array}{l}\text { Spondias } \\
\text { mombin }\end{array}$ & 75 & 4.20 & 36 & 4.15 & 16.50 & 5.20 & 13.55 \\
\hline 5 & Inga punctata & 92 & 5.15 & 48 & 5.53 & 6.53 & 2.06 & 12.74 \\
\hline 6 & Inga spectabilis & 78 & 4.36 & 45 & 5.18 & 9.36 & 2.95 & 12.50 \\
\hline 7 & $\begin{array}{l}\text { Ochroma } \\
\text { pyramidale }\end{array}$ & 63 & 3.53 & 25 & 2.88 & 17.18 & 5.42 & 11.82 \\
\hline 8 & Ficus insípida & 37 & 2.07 & 29 & 3.34 & 17.93 & 5.65 & 11.07 \\
\hline 9 & $\begin{array}{l}\text { Schizolobium } \\
\text { parahyba }\end{array}$ & 65 & 3.64 & 16 & 1.84 & 14.85 & 4.68 & 10.16 \\
\hline \multirow[t]{4}{*}{10} & $\begin{array}{l}\text { Vochysia } \\
\text { guatemalensis }\end{array}$ & 93 & 5.20 & 15 & 1.73 & 8.33 & 2.63 & 9.56 \\
\hline & 102 spp. Más & 988 & 55.29 & 539 & 30.16 & 178.34 & 9.98 & 173.62 \\
\hline & Total general & 1787 & 100.00 & 868 & 100.00 & 317.15 & 100.00 & 300.00 \\
\hline & $\begin{array}{l}\text { Distrito Bahía } \\
\text { Ballena }\end{array}$ & & & & & & & \\
\hline 1 & $\begin{array}{l}\text { Vochysia } \\
\text { guatemalensis }\end{array}$ & 92 & 6.75 & 12 & 2.07 & 8.29 & 3.54 & 12.36 \\
\hline
\end{tabular}

Continúa... 
Caracterización de la flora arbórea: Bahía Ballena y Puerto Cortés

\begin{tabular}{|c|c|c|c|c|c|c|c|c|}
\hline 2 & Inga vera & 82 & 6.02 & 28 & 4.84 & 7.69 & 3.29 & 14.14 \\
\hline 3 & Inga punctata & 75 & 5.50 & 22 & 3.80 & 5.42 & 2.32 & 11.62 \\
\hline 4 & Inga spectabilis & 61 & 4.48 & 25 & 4.32 & 7.59 & 3.24 & 12.04 \\
\hline 5 & Elaeis oleiffera & 56 & 4.11 & 13 & 2.25 & 10.34 & 4.42 & 10.77 \\
\hline 6 & $\begin{array}{l}\text { Guazuma ulmi- } \\
\text { folia }\end{array}$ & 55 & 4.04 & 18 & 3.11 & 11.56 & 4.94 & 12.08 \\
\hline 7 & $\begin{array}{l}\text { Byrsonima cras- } \\
\text { sifolia }\end{array}$ & 54 & 3.96 & 11 & 1.90 & 2.45 & 1.05 & 6.91 \\
\hline 8 & $\begin{array}{l}\text { Schizolobium } \\
\text { parahyba }\end{array}$ & 44 & 3.23 & 14 & 2.42 & 12.49 & 5.33 & 10.98 \\
\hline 9 & Spondias mombin & 40 & 2.93 & 16 & 2.76 & 11.05 & 4.72 & 10.42 \\
\hline \multirow[t]{4}{*}{10} & Virola koschnyi & 38 & 2.79 & 16 & 2.76 & 9.16 & 3.91 & 9.46 \\
\hline & 92 spp. Más & 766 & 56.20 & 404 & 69.78 & 234.14 & 17.18 & 143.15 \\
\hline & Total general & 1363 & 100.00 & 579 & 100.00 & 234.14 & 100.00 & 300.00 \\
\hline & $\begin{array}{l}\text { Distrito Puerto } \\
\text { Cortés }\end{array}$ & & & & & & & \\
\hline 1 & $\begin{array}{l}\text { Guazuma ulmi- } \\
\text { folia }\end{array}$ & 48 & 11.32 & 15 & 8.11 & 4.15 & 5.00 & 24.43 \\
\hline 2 & Spondias mombin & 35 & 8.25 & 16 & 8.65 & 5.45 & 6.57 & 23.47 \\
\hline 3 & Elaeis oleiffera & 31 & 7.31 & 7 & 3.78 & 11.04 & 13.30 & 24.40 \\
\hline 4 & $\begin{array}{l}\text { Ochroma pyrami- } \\
\text { dale }\end{array}$ & 25 & 5.90 & 8 & 4.32 & 5.01 & 6.04 & 16.26 \\
\hline 5 & Inga vera & 24 & 5.66 & 13 & 7.03 & 3.34 & 4.02 & 16.71 \\
\hline 6 & Cocos nucifera & 21 & 4.95 & 5 & 2.70 & 2.06 & 2.48 & 10.13 \\
\hline 7 & $\begin{array}{l}\text { Schizolobium } \\
\text { parahyba }\end{array}$ & 21 & 4.95 & 2 & 1.08 & 2.36 & 2.85 & 8.88 \\
\hline 8 & Cordia bicolor & 19 & 4.48 & 7 & 3.78 & 1.64 & 1.97 & 10.24 \\
\hline 9 & $\begin{array}{l}\text { Trema integérri- } \\
\text { ma }\end{array}$ & 18 & 4.25 & 3 & 1.62 & 0.84 & 1.02 & 6.88 \\
\hline \multirow[t]{3}{*}{10} & Inga spectabilis & 17 & 4.01 & 7 & 3.78 & 1.77 & 2.13 & 9.92 \\
\hline & 42 spp. Más & 165 & 38.92 & 102 & 55.14 & 45.35 & 54.63 & 148.68 \\
\hline & Total general & 424 & 100.00 & 185 & 100.00 & 83.01 & 100.00 & 300.00 \\
\hline
\end{tabular}

Fuente. Juan Carlos Villegas, 2017 
La curva de acumulación de especies muestra que con 70 muestras de las 156 realizadas se alcanzó $100 \%$ de las especies representadas en el área de estudio (ver figura 4 ).

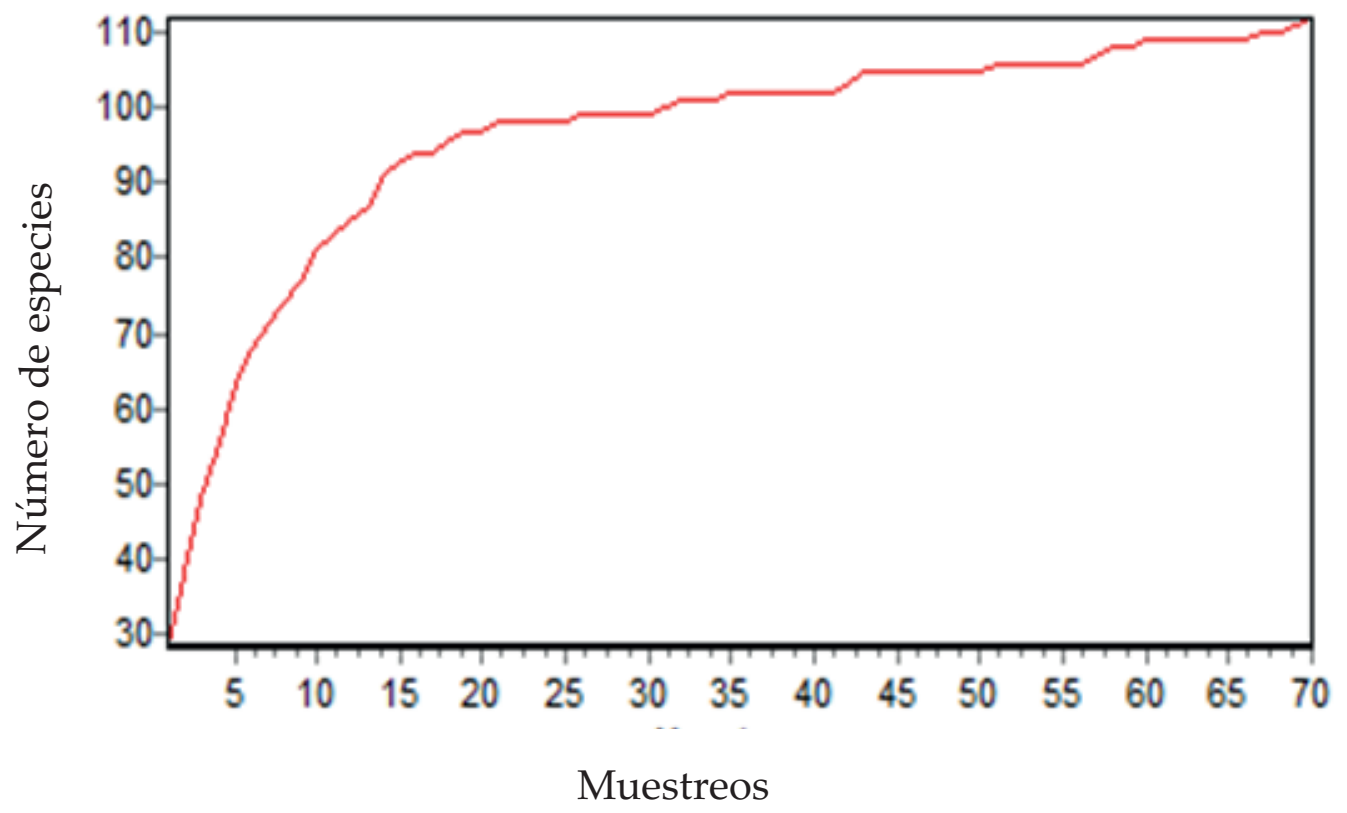

Figura 4. Acumulación de especies por parcelas demostrativas en el área de estudio, Osa 2017. Fuente: Juan Carlos Villegas, 2017.

\section{Discusión}

Estructura horizontal de los bosques delimitados en la zona costera de los distritos Bahía Ballena y Puerto Cortés.

Durante la presente investigación se identificaron en el área de estudio, según el esfuerzo de muestreo, 112 especies de árboles, para una densidad de $14.35 \mathrm{sp} / \mathrm{ha}$. En el caso de Costa Rica, se han estimado valores que oscilan entre 20 y 100 especies arbóreas/ha. Las zonas de vida muy húmedas basales y premontanas, tales como las ubicadas en la Península de Osa o el Valle Escondido, son las más diversas (Di Stefano et al., 1995).
La cantidad de árboles identificados por hectárea sugierevaloresinferioresaotros registros para esta misma zona, Morales y colaboradores (2012) registraron valores mínimos y máximos entre los 362 y 1408 Ind/ha según la edad de los bosques. Para la presente investigación, los mayores registros se obtienen de los bosques en Puerto Cortés. Para Morales et al. (2012), la edad de los bosques en Osa repercuten en un mejor estado de conservación y con mayores estadios de recuperación, a partir de una mayor cantidad de individuos por hectárea.

No obstante, la investigación de Morales et al. (2012) consideró árboles con DAP a partir de los $5 \mathrm{~cm}$, mientras que en esta investigación se registraron los árboles superiores a $15 \mathrm{~cm}$ DAP, 
lo cual evidentemente deja una población de muestreonoconsiderada.Asuvez,lainvestigación de Morales et al. (2012) incluyó bosques en una gran área protegida de la Península de Osa, la Reserva Forestal Golfo Dulce en los sectores de Mogos, Santa Cecilia, Piro y Matapalo, bosques con un fuerte estatus de conservación, no comparable con los bosques de la zona costera en Bahía Ballena y Puerto Cortés, con evidente mayor alteración e impacto por la tala.

Por su parte Berry (2002) menciona registros de entre 618 y $654 \mathrm{Ind} / \mathrm{ha}$ para la región de Manaos en la Amazonia central, y de 675 Ind/ ha. en el Chocó del Pacífico colombiano, también con una población muestreada a partir de los 10 $\mathrm{cm}$. DAP.

Un registro de valores de entre $218.08 \mathrm{Ind} / \mathrm{ha}$, y 273.5 Ind/ha. para esta zona de estudio podría considerarse como alto para bosques con gran presión antropogénica por la matriz ecosistémica y el agropaisaje en el cual se encuentran (Morera 2010), además de la edad de la población cuantificada $(<15 \mathrm{~cm}$. DAP).

Lo anterior se puede reafirmar al considerar que la población muestreada no consideró la clase diamétrica inferior a los $10 \mathrm{~cm}$. DAP, lo cual podría representar entre 52 y $58 \%$ de la totalidad de los registros de acuerdo con Cascante y Estrada (2001); o como lo sugirieron Tenorio y colaboradores (2009) para su investigación en bosques de bajura de la zona norte en Costa Rica: la totalidad de individuos por hectárea no superó los 367.55.

Para Tenorio et al. (2009), la mayor cantidad de individuos se registran en la clase diamétrica inferior a los $10 \mathrm{~cm}$. DAP dados los muchísimos árboles que pueden establecerse durante los primeros años; sin embargo, conforme aumenta la clase diamétrica, la cantidad de individuos disminuye producto de la competencia y las exigencias lumínicas que requieren algunas especies para mantenerse dentro del bosque. Por ende, se produce alta mortalidad de individuos en las especies heliófitas, dando paso en la sucesión ecológica a especies de mayor longevidad, con menores necesidades de luz.

En esta investigación, la distribución de los individuos por clases diamétricas sugiere una conformación en "J" invertida. Se registra la mayor cantidad de individuos superiores a 15 $\mathrm{cm}$. DAP en esta categoría.

Para Morales et al. (2012), en un área determinada pueden existir más individuos pequeños que grandes; por ende, en la medida que los árboles sean de menor porte, mayor será su abundancia por unidad de área y mayor será la riqueza con respecto a clases de tamaño mayores. Esta "J" invertida donde se registra la mayoría de árboles y especies en las primeras clases diamétricas, asegura el equilibrio de los bosques (primarios y secundarios).

De acuerdo con Finegan (1997), la sucesión ecológica de los bosques presenta tres etapas: la primera está dominada por hierbas y arbustos, normalmente encontrada en el sotobosque; luego, por el banco de semillas, por la dispersión y por otros factores, se establecen los árboles pioneros o heliófitos efímeros, donde ubicamos las clases diamétricas inferiores; siguen, consecuentemente, los árboles de vida más larga como las heliófitas durables, las cuales según su especie se irán ubicando en las clases diamétricas superiores, menos abundantes.

Consistente con la interpretación de la conformación de "J" invertida, el índice de valor de importancia (IVI) arrojó los mayores registros para especies heliófitas efímeras como las guabillas (Inga sp.), el jobo (Spondias mombin), el guácimo (Guazuma ulmifolia) y el balso (Ochroma pyramidale), locual sugierela presencia debosques en tempranos estadios de sucesión ecológica. 
Estructura vertical de los bosques en el área de estudio.

La estructura vertical de un bosque está determinada por la distribución de los organismos, en este caso de los árboles a lo alto de su perfil; además, estará en función de las características biológicas que para las especies son determinantes en su crecimiento, especialmente por la absorción de luz (Louman, Quirós y Nilsson, 2001). Richards (1996) definió cinco estratos en los bosques tropicales: los árboles emergentes, el dosel, el subdosel, el sotobosque arbustivo y el sotobosque herbáceo.

Según Louman et al. (2001) la estratificación vertical del bosque es importante en la determinación de microclimas, que incide en factores como la luz y los cambios atmosféricos. Por consiguiente, el entendimiento de tal estructura y la composición del bosque en distintas alturas sobre el nivel del suelo es importante a fin de establecer su estadio de crecimiento o nivel de madurez.

Las especies de árboles que presentaron individuos con alturas superiores fueron la ceiba, el terciopelo o pica pica, el aguacatillo y el baco, todas especies heliófitas duraderas, pero con poca representación en los muestreos.

La mayoría de los árboles presentaron alturas bajas o intermedias entre 5 y 15 metros. Lo anterior sugiere, de manera consistente con los datos de DAP, sus áreas basales y la distribución diamétrica, que dichos bosques son jóvenes y en estado de regeneración incipiente.

Los registros para bosques de la Península de Osa sugieren que para bosques con no menos de 15 años de regeneración, sus alturas promedio no sobrepasan los 17 metros; mientras que en cuanto a bosques con más de 30 años de edad, sus alturas promedio alcanzan los 29 metros (Morales 2010), lo que podemos considerar como un buen parámetro para estimar la edad de estos bosques en la zona de estudio.

\section{Conclusión}

Los bosques estudiados en la zona costera de los distritos Bahía Ballena y Puerto Cortés en el cantón de Osa, en la zona sur de Costa Rica, pueden considerarse bosques muy ricos en especies. Esto reafirma la condición de alta biodiversidad para estos bosques ubicados en la zona de vida muy húmeda basal.

A su vez, no solo la riqueza de especies sugiere la elevada biodiversidad arbórea en esta zona costera, sino la cantidad de árboles que se encuentran en ella, donde se registraron altas densidades. Se evidencia, pues que son bosques compactos y muy densos, con gran cantidad de especies y de individuos.

No obstante, los análisis sugieren que son bosques jóvenes o con constante perturbación, cuyas causas exceden el presente análisis, pero que podrían estar presentes, sean naturales o antrópicas. Los tempranos estadios de regeneración indicaría la distribución diamétrica de los árboles y sus alturas. Se encontró muchos árboles con diámetros bajos y con bajas alturas a su vez.

Finalmente, la composición de la diversidad arbórea también sugiere esta condición de temprana regeneración, con el registro (en su mayoría) de especies heliófitas efímeras, cuya abundancia y dominancia demuestra el gran dinamismo de esos bosques. Muy pocas especies presentaron diámetros elevados, y solo un individuo de una especie superó los 30 metros de altura. Por consiguiente, podemos concluir que estos bosques son jóvenes y están en un proceso dinámico de recuperación.

El distrito de Bahía Ballena registró más cantidad de bosques $\mathrm{y}$, por ende, presenta la más riqueza forestal en la zona costera. No así el distrito de Puerto Cortés, cuya riqueza y 
cantidad de bosques es más escasa. Esto se refleja por la gran expansión de la frontera agrícola en este distrito.

Es recomendable que en los procesos de planificación y ordenamiento territorial en el distritodeBahíaBallenaseconsiderentalesparches de bosque, especialmente en lo que concierne a la zona marítimo-terrestre, en razón de que constituyen una rica fuente de recursos naturales que conlleva un atractivo turístico crucial.

Dichos bosques aportan belleza escénica a este distrito y alternativas de desarrollo amigables con el medio ambiente, particularmente si tales recursos naturales son considerados por las autoridades ambientales para el ordenamiento territorial. Son, por lo tanto, susceptibles de incorporación al régimen del Patrimonio Natural del Estado, que conlleva un uso con condiciones especiales a partir de la legislación forestal de Costa Rica.

A partir de los registros y análisis desarrollados, se concluye que, efectivamente, la principal diversidad de bosques y elementos arbóreos de la biodiversidad se encuentran en el distrito de Bahía Ballena, no así en Puerto Cortés, donde estos recursos ya se han diezmado mucho.

\section{Agradecimientos}

A la Universidad Nacional (UNA) por permitirme desarrollar este proyecto de estudios doctorales en su programa de Ciencias Naturales para el Desarrollo (DOCINADE); y al Área de Conservación Osa (ACOSA) del Sistema Nacional de Áreas de Conservación (SINAC) por brindar los permisos para esta investigación y el apoyo técnico de sus funcionarios.

\section{Referencias}

Armenteras, D. y Rodríguez, N. (2014). Dinámicas y causas de deforestación en bosques de Latinoamérica: una revisión desde 1990. Rev. Colom. Forest. 17(2), 233-246.
Balzarini, M.G., Casanoves, F., Di Rienzo J.A., González, L.A., Robledo, C.W. y Tablada, E.M. (2017). InfoStat, versión 1,1, Manual del Usuario. Grupo InfoStat, FCA, Universidad Nacional de Córdoba.. : Córdoba, Argentina: Editorial Brujas.

Barquero, V. A. y Jiménez-Hernández, F. (2009). Diversidad y distribución de palmas (Arecaceae) en tres fragmentos de bosque muy húmedo en Costa Rica. Rev. Biol. Trop. 57(1), 83-92.

Barrantes, M. P. (2013). Costa Rica, sin ingredientes artificiales: El rol del Estado en la expansión del turismo residencial en las zonas costeras. Anuar. Est. Centroam. 39, 233-261.

Berry, P. E. (2002). Diversidad y endemismo en los bosques neotropicales de bajura. Capitulo 4. En: Ecología y conservación de bosque neotropicales. Guariguata, M. R. y Kattan, G.H. (Eds.).Texas: Universidad de Texas..

Cascante, A. y Estrada, A. (2001). Composición florística y estructura de un bosque húmedo premontano en el Valle Central de Costa Rica. Rev. Biol. Trop., 49(1), 213-225.

Cayuela, L., Golicher, D. J., Rey, J. M., González-Espinoza, M. \& Ramírez-Marcial, N. (2006). Fragmentation, disturbance and tree diversity conservation in tropical montane forests. J. Applied Ecol. 43, 1172-1181.

Celentano, D.; Zahawi, R. A.; Finegan, B.; Casanoves, F.; Osterta, R.; Cole, R. J. y Holl, K. D. (2011, setiembre). Restauración ecológica de bosques tropicales en Costa Rica: efecto de varios modelos en la producción, acumulación y descomposición de hojarasca. Rev. Biol. Trop. (Int. J. Trop. Biol. ISSN-0034-7744). 59(3), 1323-1336.

Cook, E., Briffa, K., Shiyatov, S., Mazepa, V., \& Jones, P.D. (1992). Data analysis. Cook, E. \& Kairiukstis, L. (Eds.). Methods of dendro- 
chronology: applications in the environmental sciences (97-162). Dordrecht: Kluwer Academic Publishers.

Curtis, J. T. \& McIntosh, R. P. (1951). An Upland Forest Continuum in the Prairie-Forest Border Region of Wisconsin. Ecology. 32(3), 476-496.

Dirzo, R., Broadbent, E.N., Almeyda, A.M., Morales, L., Almeyda, S. y Quispe, C.A. (2014, julio). Ecosistemas terrestres de la región de Osa y Golfito, Costa Rica. San José, Costa Rica: Woods Institute for the Environment, Stanford University.

Di Stéfano, J. F., Brenes, L. y Mora, V. (1995). Composición florística y estructura de un bosque primario del piso premontano pluvial, en San Ramón, Costa Rica. Rev. Biol. Trop., 43(13), 67-73.

Di Stéfano, J. F.; Nielsen, V.; Hoomans, J. y Fournier, L. A. (1996). Regeneración de la vegetación arbórea en una pequeña reserva forestal urbana del nivel premontano húmedo, Costa Rica. Rev. Biol. Trop, 44(2), 575-580.

Ferraz, G., Russell, G.J., Stouffer, P.C., Bierregaard, R.O. Jr., Pimm, S.L. \& Lovejoy, T.E. (2003). Rates of species loss from Amazonian forest fragments. Proc. Nation. Acad. Sci. 100, 14069-14073.

Finegan, B. (1997). Bases ecológicas para el manejo de bosques secundarios de las zonas Húmedas del trópico americano, recuperación de la biodiversidad y producción sostenible de madera. Memorias del “Taller Internacional sobre el estado actual y potencial de manejo y desarrollo del bosque secundario Tropical en América latina. Puca11 pa.

Fowler, J., Cohen, L., \& Jarvis, P. (1998). Practical statistics for field biology. (2da. ed.). West Sussex, England: John Wiley \& Sons.

Galanes, I. \& Thomlinson, J. (2009). Relationships between spatial configuration of tropical forest patches and woody plant diversi- ty in northeastern Puerto Rico. Plant Ecology 201, 101-113.

Hammel, B. E., Zamora., N. y Grayum, M. H. (Eds.). (1998). Manual de Plantas de Costa Rica. Vol. 1: Monocotiledóneas. Vol. 2: "Dicotiledóneas" (Acanthaceae-Euphorbiaceae). Vol. 3: "Dicotiledóneas" (Fabaceae-Myrtaceae). Vol. 4: "Dicotiledóneas" (Nyctaginaceae-Zygophyllaceae) y "Gimnospermas". Missouri Bot. Gard. Press, St. Louis (manuscript).

Harris, L.D. (1984) The Fragmented Forest: Island Biogeography Theory and the Preservation of Biotic Diversity. Chicago: University of Chicago Press.

Hill, J.L. \& Curran, P.J. (2003). Area, shape and isolation of tropical forest fragments: effects on tree species diversity and implications for conservation. J. Biogeog. 30, 1391-1403.

Henderson, P. A. \& R. M. Seaby. (2006). Species, diversity and richness. Versión 4.0 Pisces Conservation Ltda. (Programa informático). Reino Unido: Pennington, Lymington.

Hernández, Z. (2000, 11 de febrero). Cronosecuencia del bosque seco secundario tropical en el Parque Nacional Palo Verde, Bagaces, Costa Rica. Seminario Avances en el Manejo del Bosque Secundario en Costa Rica.. Pavas, San José (Costa Rica) (No. 634.92097286 S471 2000). Cartago, Costa Rica: Tecnológico de Costa Rica, Escuela de Ingeniería Forestal Proyecto COSEFORMA/GTZ.

Jiménez-Valverde, A. y Hortal, J. (2003). Las curvas de acumulación de especies y la necesidad de evaluar la calidad de los inventarios biológicos. Rev. Ibér. Aracn. 8(31), 151-161.

Kappelle, M; Castro, M.; Acevedo, H; González, L. y Monge, H. (2002). Ecosistemas del Área de Conservación Osa. Santo Domingo de Heredia, Costa Rica: INBio.. 
Lamprecht, H. (1990). Silvicultura en los trópicos: Los ecosistemas forestales y sus especies arbóreas; posibilidades y métodos para un aprovechamiento sostenido. Alemania: GTZ.

Laurance, W.F. \& Bierregaard, R.O. Jr. (1997). Tropical Forest Remnants: Ecology, Management and Conservation of Fragmented Communities. Chicago: University of Chicago Press.

Leiva, J. A., Rocha, O. J., Mata, R., y Gutiérrez-Soto, M. V. (2009). Cronología de la regeneración del bosque tropical seco en Santa Rosa, Guanacaste, Costa Rica. II. La vegetación en relación con el suelo. Rev. Biol. Trop. 57(3), 817-836.

Louman, B; Quirós, D. y Nilsson, M. (2001). Silvicultura de bosques latifoliados tropicales con énfasis en América Central. Turrialba, Costa Rica: CATIE.

Magurran, A.E. (1988). Ecological diversity and its Measurement. New Jersey: Princeton University Press: New Jersey, 179.

Morales, M. (2010). Composición florística, estructura, muestreo diagnóstico y estado de conservación de una cronosecuencia de bosques tropicales del Corredor Biológico Osa, Costa Rica (Doctoral dissertation, tesis Lic. Cartago, Instituto Tecnológico de Costa Rica. Escuela de Ingeniería Forestal).

Morales-Salazar, M., Vílchez-Alvarado, B., Chazdon, R. L., Ortega-Gutiérrez, M., Ortíz-Malavassi, E. y Guevara-Bonilla, M. (2012). Diversidad y estructura horizontal en los bosques tropicales del Corredor Biológico de Osa, Costa Rica. Rev. Forest. Mesoam. Kurú (Costa Rica) 9(23), 19-28.

Morera, C. (2010). Estructura del paisaje y desarrollo turístico en la Fila Brunqueña, Costa Rica. Rev. Geog. Amer. Cent. 44(1), 91-108.

Morera, B. C., Sandoval, M., y Pintó, J. (2018, marzo). Transformaciones espacio-tempo- rales de la cobertura vegetal en el Parque Nacional Corcovado, 1960-2014. Rev. Biol. Trop. 66(1), 352-367.

Moreno, C. E. (2001). Métodos para medir la biodiversidad, Vol. 1. Zaragoza, España: M\&T-Manuales y Tesis SEA.

Oviedo, P. E., Alvarado, A. y Fournier, L. (2009). Caracterización de la vegetación alrededor de los senderos del sector Quebrada González, Parque Nacional Braulio Carrillo, Costa Rica. Rev. Uniciencia 23, 41-49.

Pincheira-Ulbrich, J., Rau, J. R. y Peña-Cortés, F. (2009). Tamaño y forma de fragmentos de bosque y su relación con la riqueza de especies de árboles y arbustos. Fyton. 78, 121128.

Richards, P. W. (1996). The tropical rain forest: an ecological study (2.a Ed. Con correcciones). Reino Unido: Cambridge University Press.

Rosenblatt, D.L., Heske, E.J., Nelson, S.L., Barber, D.M., Miller, M.A. \& MacAllister, B. (1999). Forest fragmentation in east-central Illinois: islands or habitat patches for mammals? Amer. Midland Natur. 141, 115-123.

Sánchez, D., Harvey, C. A., Grijalva, A., Medina, A., Vílchez, S. y Hernández, B. (2005). Diversidad, composición y estructura de la vegetación en un agropaisaje ganadero en Matiguás, Nicaragua. Rev. Biol. Trop. 53(34), 387-414.

Saunders, D., R. Hobbs \& C. Margules (2001). Biological consequences of ecosystem fragmentation: a review. Conservation Biology 5, 18-32.

Sistema Nacional de Áreas de Conservación (SINAC). Gobierno de Costa Rica. (2015). Inventario Nacional Forestal de Costa Rica 2014-2015. Resultados y caracterización de los recursos forestales. Preparado por: Emanue1li, P., Milla, F., Duarte, E., Emanuelli, J. y 
Chavaría, M.I. San José, Costa Rica: Programa REDD/CCAD/GIZ, SINAC. .

Sokal, R. \& Rohlf, J. (1981). Biometry. Freeman and company. Estados Unidos, New York, .

Solow, A.R. (1993). A simple test for change in community structure. J. Anim. Ecol. 62(1),191-193.

Tenorio, C., Solano-Durán, J y Castillo-Ugalde, M. (2009). Evaluación de la composición florística y estructural en un bosque primario intervenido en la zona norte de Costa Rica. Rev. Forest. Mesoam. Kurú (Costa Rica) 6(16), 52-62.

Vargas-Fonseca, L. (2012). Análisis de una cronosecuencia de bosques tropicales del corredor biológico Osa, Costa Rica. Tesis de licencia- tura. Cartago, Costa Rica: Instituto Tecnológico de Costa Rica, Escuela de Ingeniería Forestal.

Villegas, J. C. (2017). Clasificación de ecosistemas objeto de conservación en la zona costera del pacífico sur de Costa Rica. Rev. Posg. Soc. 15(2), 1-19.

Windevoxhel, N., Rodríguez, J., y Lahmann, E. (2003). Situación del manejo integrado de zonas costeras en Centroamérica: Experiencias del Programa de Conservación $\mathrm{Hu}$ medales y Zonas Costeras de UICN para la región. SF 28, 3-40.

Woodson, R.E. Jr. \& Schery, R.W. (1980). Flora of Panama. Annals of the Missouri Botanical Garden. 67(4).
Recibido: 07 de setiembre de 2017 Aceptado: 04 de abril de 2018 